\title{
User Fairness and QoS Aware based Effective Resource Allocation for Downlink NOMA Cellular Systems
}

PRIYA GUPTA ( $\nabla$ pgupta@ec.iitr.ac.in )

Indian Institute of Technology Roorkee https://orcid.org/0000-0003-0864-5113

D. Ghosh

Indian Institute of Technology Roorkee

\section{Research Article}

Keywords: 5G Cellular Network, System Throughput, NOMA, SIC, Resource allocation.

Posted Date: February 11th, 2022

DOI: https://doi.org/10.21203/rs.3.rs-1306140/v1

License: (c) (i) This work is licensed under a Creative Commons Attribution 4.0 International License.

Read Full License 


\title{
User Fairness and QoS Aware based Effective Resource Allocation for Downlink NOMA Cellular Systems
}

\author{
Priya Gupta • D. Ghosh
}

Received: date / Accepted: date

\begin{abstract}
Non orthogonal multiple access (NOMA) is expected to be the most significant and viable solution for fifth generation $(5 \mathrm{G})$ communication system due to its incredible advantage of spectral efficiency. This paper proposes joint channel allocation and power optimization for downlink NOMA system, so as to maximize the system throughput while preserving the minimum data rate of every user equipment necessary to maintain quality of service and successive interference cancellation constraint. The optimization problem so formulated is a joint mixed integer nonlinear programming problem. Hence, two stage solution is proposed that uses mosek solver for channel assignment followed by power allocation across assigned channels. We use the difference between two convex functions (DC) programming technique for modifying the nonconvex optimization problem into a convex subproblem. Thus, we achieve efficient and fair power allocation coefficients across channels by solving the convex subproblem iteratively. Simulation results demonstrate better efficiency of our proposed scheme for resource allocation compared to some of the existing baseline schemes.
\end{abstract}

Keywords 5 G Cellular Network, System Throughput, NOMA, SIC, Resource allocation.

Priya Gupta

Department of Electronics and Communication, Indian Institute of Technology Roorkee, Roorkee, Haridwar, Uttarakhand, India

E-mail: pgupta@ec.iitr.ac.in

D. Ghosh

Department of Electronics and Communication, Indian Institute of Technology Roorkee, Roorkee, Haridwar, Uttarakhand, India

E-mail: debashis.ghosh@ece.iitr.ac.in 


\section{Introduction}

The multiple access techniques play a significant role in wireless communication networks. The conventional multiple access schemes had been good enough to serve users with low data rates for the last two decades. However, the exponential growth of data traffic is expected to result in demand for high data rate within limited resources. For solving this issue, it is recommended to switch from orthogonal multiple access (OMA) to non-orthogonal multiple access (NOMA) technique. Till now, NOMA scheme is less investigated by researchers of the industry as well as academia. Although, OMA scheme is good enough to obtain spectral efficiency (SE) for single-user detection, it is to perform better in multiuser scenario [1,2]. NOMA system has been attracted considerable attention and is envisioned for the use in $5 \mathrm{G}$ communication system due to its better throughput performance. $5 \mathrm{G}$ demands higher data rate, low latency and massive connectivity due to their real time applications. Since, the orthogonal resource allocation scheme (ORA) fails to provide sufficient data rate to each requested user due to tremendous resource consumption, non orthogonal resource allocation (NORA) scheme comes into play. NOMA turn out to be the best suitable candidate for the next generation communication [3]. Although ORA reduces the chances of channel interference, it is unable to serve each user due to limited resources. On the other hand due to good interference handling capability, NOMA is appreciated for its higher SE as compared to OMA in the scarce resource situation. This advantage does not in any way compromise user fairness facilitated by successive interference cancellation (SIC) in NOMA 4. Superposition code (SC) at the transmitter side and SIC at the receiver side, together help in reducing the channel interference up to some extent [5].

Controlling the interference gives a significant improvement in system performance, and this is possible by smart resource allocation. Therefore, efficient resource allocation plays a key role in managing the interfering channel and helps in improving the system performance. Accordingly, some existing literature survey on NOMA cellular system focus on resource allocation. A greedy channel assignment scheme and power allocation algorithm for NOMA system is proposed in [6] that employs Difference of convex (DC) programming scheme for power allocation in a multicell scenario.

In 7], a cooperative NOMA system is investigated for better SE where users have prior information about the channel. Sequential quadratic transform (SQP) 23 technique is exploited as non-convex optimization that provides reliable solution for better SE. Authors in 8 discussed the power minimization problem in IoT devices using NOMA system, proposed SQP based techniques for power allocation and compared their results with that of Karush Kuhn Tucker (KKT) method. They modeled nonlinear programming problem into quadratic programming sub-problem by exploiting the SQP approach. However, fairness in resource allocation was not measured in terms of Jain Fairness Index (JFI). The authors in $[9]$ proposed interior point method (IPM) 23 for power allocation and used fmincon solver available in MATLAB. Their 
work considered only quality of service (QoS) constraint in maximizing the sum-rate while ignoring the SIC constraint. Since the SIC constraint is essential for developing NOMA system, this leaves room for further improvement over the work proposed in 9].

Some other literature discusses Alamouti codes for cluster-based cell in downlink NOMA where the target is to provide sufficient data rate to far users without sacrificing data rate of the near users $[10$. In $[11$, the impact of user pairing for efficient SE in fixed cooperative NOMA systems is discussed where the dynamic user pairing approach limited to two users is employed. This pairing/grouping is totally based on the channel condition. Authors in 12, 13 discussed suboptimal resource allocation scheme for maximizing sumrate capacity while considering only the power budget and minimum data rate constraint. In 12], users are clustered and sorted on the basis of their channel variance to achieve the ergodic rate for multiple input multiple output (MIMO) NOMA system. In [13], users are grouped on the basis of QoS without considering the SIC constraint in multiuser MIMO NOMA network.

In 14], energy efficient NOMA network is designed for efficient resource allocation using game theory for optimal power assignment and user scheduling. The joint optimization herein is nonconvex and NP-hard. In the view of this, the combined problems of channel matching and power assignment problem are taken care of resolved by decoupling the two problems. Moreover, the proposed work is limited to two users, and the results are compared with fractional transmit power allocation (FTPA) 26 scheme that does not consider user fairness. Authors in 15 maximized the system sum-rate for Okumura Hata model [1], satisfying Jain's fairness constraint. However, the computation complexity of the suggested scheme is high due to large feasible search area of fair power. The study in 16 investigates system throughput maximization for both uplink and downlink NOMA network. The formulated problem is solved by user clustering and power allocation. Moreover, the KKT condition is used for optimal resource allocation in case of both uplink and downlink scenarios. In [17], NOMA system is investigated under two scenarios; the first one is with instantaneous channel state information (CSI) and the second is with the average CSI. In addition, resource allocation is done while maintaining user fairness and NOMA results are compared with the conventional scheme. NOMA system throughput and weighted sum-rate maximization are discussed in [18] under optimal KKT conditions. Furthermore, energy efficiency (EE) and weighted EE are maximized for the same NOMA system but limited to two users only to avoid system's high complexity system configuration. Authors in 19] applied Hungarian (HNG) algorithm so that users can be arranged in a cluster and then KKT condition is applied for obtaining optimal power allocation to those clustered users. Sumrate maximization is performed using the scheme discussed above. However, the limitation of the scheme is that only two users are paired on the same channel. In 20 also, only two-user scenario is considered so as to avoid complicating the problem while handling only two constraints (power budget and minimum data rate). Reference [21], also discusses NOMA system model under two-user scenario 
TABLE 1

\begin{tabular}{|c|c|c|c|c|c|c|c|c|}
\hline \multirow{2}{*}{ Ref } & \multicolumn{3}{|c|}{ Constraints } & \multicolumn{2}{|c|}{ Users considered on each channel } & \multicolumn{2}{|c|}{ Optimized variable } & \multirow{2}{*}{ Method used } \\
\hline & power budget & Rmin & SIC & two users & more than two users & power & channel & \\
\hline 17 & 1 & 1 & $x$ & $x$ & $d$ & 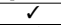 & $x$ & Min-max \\
\hline$\overline{19}$ & $\checkmark$ & $\checkmark$ & $x$ & $\checkmark$ & $x$ & $\checkmark$ & 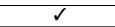 & HNG+KKT \\
\hline 20 & $\mathrm{~s}$ & 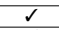 & $x$ & $\checkmark$ & $x$ & 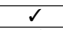 & $x$ & Equal power and KKT \\
\hline$\frac{20}{21}$ & 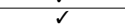 & $\sqrt{1}$ & $x$ & 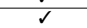 & $x$ & $\mathrm{~d}$ & $x$ & Equal and proportional power \\
\hline$-2 \overline{22}$ & $\checkmark$ & 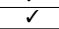 & $x$ & $\checkmark$ & $x$ & $\sqrt{1}$ & $d$ & HNG+fmincon solver \\
\hline Proposed & $\checkmark$ & $\checkmark$ & $\checkmark$ & $x$ & $\checkmark$ & $\checkmark$ & 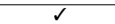 & mosek+DC \\
\hline
\end{tabular}

with power budget and minimum data rate constraints only. In 22 , along with downlink scenario, uplink NOMA system model is also investigated where optimal resource allocation is applied but without the SIC constraint. Table 1 lists some of the major developments in resource allocation in NOMA system along with their respective research gaps that motivates us to do research on user fairness and QoS aware-based effective resource allocation for downlink NOMA cellular systems, as proposed in this paper.

The ever increasing scarcity of resources has always motivated researchers to optimize the resource allocation schemes. Since, exponential growth in the number of users demands more throughput, different scheme for resource allocation need to be developed and subsequently verified by fairness index. Therefore, in this paper, we aim to develop a strategy for joint optimal channel assignment and power allocation. The novelty of this work is in finding fair power allocation coefficient for the channels assigned to the users in a NOMA system. The efficacy of the distribution is evaluated in terms of system throughput.

In a nutshell, the major contributions of our proposed work in this paper are as follows.

- From the literature survey, we see that the maximum number of users sharing the same channel considered in the existing literature is restricted to two which in reality generally not the case. Therefore, we propose to generalize the resource allocation algorithm where more than two users can be multiplexed on the same resource.

- To the best of our knowledge, no scheme for resource optimization in such a scenario is available in the literature. Apart from this, by adjusting QoS (minimum data rate constraint) and SIC constraints, the proposed resource allocation scheme performs better in terms of fairness. The formulated problem is a mixed integer non-linear programming (MINLP) problem and hence NP-hard due to its combinatorial nature. We propose efficient algorithm for finding the solution to the NP-hard non-convex joint resource allocation problem.

\section{Downlink Transmission System Environment and Problem formulation}

We consider a downlink NOMA system, where channel gain of each user is different from the each other. The base station (BS) transmits the superposed 
signal non-orthogonally to the multiplexed users on the same radio resource. This superposed signal at the receiver consists of multiplexing of users' signals with different power level which is crucial. Therefore, it can be handled by NOMA technique. The strongest user (having the highest channel gain) performs SIC first, subtracts the interfering signal from the superposed signal and after that decodes its own signal. Although the strongest user experiences highest interference due to high power allocation to weaker user, strong user first performs SIC and then subtracts the interfering signal. Finally, decode its own signal. Similarly, the weakest user (having the lowest channel gain) experiences low interference due to low power allocation to stronger user. Consequently, NOMA system can easily suppress the interfering signals.

Consider three user equipment $(U E)$ downlink NOMA scenario where the transmitter is BS and the receiver is $U E$. The desired signals and gains of the $U E \mathrm{~s}$ are $x_{1}, x_{2}, x_{3}$ and $h_{1}, h_{2}, h_{3}$, respectively. If the channel gains are ordered as $h_{1}>h_{2}>h_{3}, U E_{1}$ has the highest channel gain than others. Then $U E_{1}$ performs SIC and cancels the effect of interference from the remaining users whereas $U E_{2}$ is unable to cancel the interference effect from $U E_{1}$ but capable of cancelling the interference effect of $U E_{3}$.

We consider the downlink NOMA system model where the BS is centered in the cellular circular region and serving users that are uniformly distributed within the cell as illustrated in Fig1. It is assumed that all users are active. Let, there be multiple $U E \mathrm{~s}$, indexed as $u=1,2, \ldots,|\mathcal{U}|$, where $|\mathcal{U}|$ is the number of $U E$ s. There are $|\mathcal{C}|$ number of channels, indexed as $c=1,2, \ldots,|\mathcal{C}|$, but each $U E$ is allowed to occupy at most one channel only. According to the current scenario (large data traffic and less resource), we assume that the cell is fully occupied by the users and free channels is not available. That is, the number of channels is less than number of users, i.e. $|\mathcal{C}|<|\mathcal{U}|$. The bandwidth of each channel is given as $B_{c}=\frac{B}{|\mathcal{C}|}$, where $B$ is the total available bandwidth.

It is assumed that channel assignment in the NOMA network is centralized and the BS is responsible for supervising this operation. Therefore, it is necessary that the entire CSI be available at the BS, i.e., the BS is aware of all channel information. By exploiting the downlink NOMA protocol, we may derive the BS transmitted superposed signal for the $u$ th user on the $c$ th channel as

$$
\sum_{u=1}^{|\mathcal{U}|} \sqrt{a_{c, u} p_{c}} s_{u}
$$

where $s_{u}$ represents the symbol of the $u$ th user, $p_{c}$ is the power on each channel and $a_{c, u}$ is the power allocation coefficient of $U E_{c, u}$. Then the received signal at $U E_{c, u}$ is given as

$$
\sqrt{a_{c, u} p_{c}} h_{c, u} s_{u}+\sum_{v=1, v \neq u}^{|\mathcal{U}|} \sqrt{a_{c, v} p_{c}} h_{c, u} s_{v}+z_{c, u}
$$




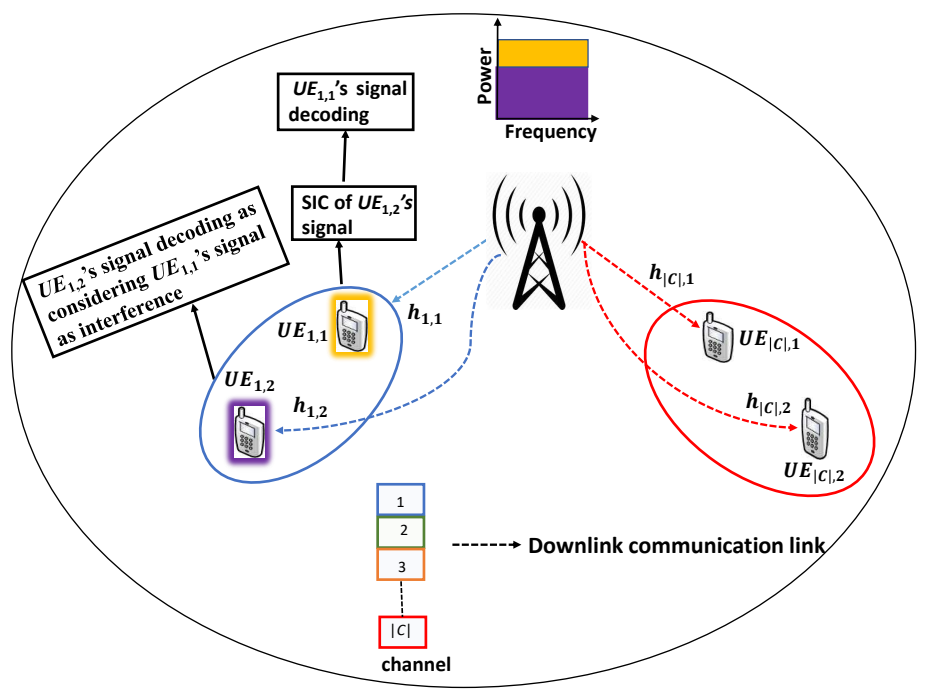

Fig. 1. Interference scenario in single cell (downlink sharing).

where $h_{c, u}$ is the channel gain given as $h_{c, u}=g_{c, u} d_{u}{ }^{-\zeta}, g_{c, u}$ is the coefficient of channel gain from the BS to $U E$ that follows Rayleigh distribution, $d_{u}$ is the distance of the $u$ th $U E$ from the BS and $\zeta$ is the path loss exponent. The additive white Gaussian noise (AWGN) is denoted as $z_{c, u} \sim \mathcal{C N}\left(0, \sigma^{2}\right)$. It may noted that NOMA technique cannot be applied in case of symmetric channel condition. Therefore, without loss of generality we assume that each user has distinct channel gain, in the order $\left|h_{c, 1}\right|^{2}>\left|h_{c, 2}\right|^{2}>\left|h_{c, 3}\right|^{2}>\ldots,>\left|h_{c,|\mathcal{U}|}\right|^{2}$. Following Shannon's capacity formula, the data rate of the $u$ th user for the cth channel and system throughput (SR) are respectively defined as

$$
R_{c, u}\left(a_{c, u}\right)=\log _{2}\left(1+\frac{a_{c, u} p_{c}\left|h_{c, u}\right|^{2}}{\eta+\left|h_{c, u}\right|^{2} p_{c} \sum_{v=1}^{u-1} a_{c, v} \rho_{c, v}}\right)
$$




$$
\begin{array}{ccc}
S R=\max _{a_{c, u}, \rho_{c, u}}\left\{\sum_{c=1}^{|\mathcal{C}|} \sum_{u=1}^{|\mathcal{U}|} \rho_{c, u} R_{c, u}\left(a_{c, u}\right)\right\} \\
\text { subject to } \sum_{u=1}^{|\mathcal{U}|} a_{c, u}=1 & \forall c \in \mathcal{C} \\
a_{c, u} \geq 0 & \forall c \in \mathcal{C}, \forall u \in \mathcal{U} \\
\left|h_{c, u-1}\right|^{2}\left(a_{c, u}-\sum_{v=1}^{u-1} a_{c, v}\right) \geq \frac{P_{t h} \eta}{p_{c}} & \forall u \in \mathcal{U} \\
\log _{2}\left(1+\gamma_{c, u}\right) \geq R_{c, u}^{m i n} & \forall c \in \mathcal{C}, \forall u \in \mathcal{U} \\
\sum_{c=1}^{|\mathcal{C}|} \rho_{c, u}=1 & \forall u \in \mathcal{U} \\
\rho_{c, u} \in\{0,1\} & \forall c \in \mathcal{C}, \forall u \in \mathcal{U} .
\end{array}
$$

where $\eta=\sigma^{2} B$ is the noise power, $\gamma$ is the signal to interference noise ratio (SINR), and $P_{t h}$ denotes the minimum power gap between the coded and the decoded signal. $\rho_{c, u}$ denotes a binary variable that represents channel selection for the users, defined as

$$
\rho_{c, u}=\left\{\begin{array}{l}
1, \text { if } c \text { th channel is shared by the } u \text { th } U E \\
0, \text { otherwise. }
\end{array}\right.
$$

The goal of the formulated problem is to optimize the overall performance (total throughput) of the downlink NOMA network. Each downlink channel has a restriction of transmission power budget by BS, as defined in constraint (4b). The inequality defined in (4c) states that power allocation coefficient of each user is always positive, while (4d) guarantees successful SIC at the receiver . Inequality (4e) defines the minimum data rate for each $U E$. Constraints (4e) and $(4 \mathrm{~g})$ are due to channel assignment where each $U E$ is rigidly allocated at most one channel. We know that the rate is a logarithmic function, and due to the interference term in the denominator of the rate expression, our optimization problem defined in eqn (4a) is non-convex. In (4a), the SR is maximized over two discrete variables $a_{c, u}$ and $\rho_{c, u}$. Hence, the overall structure of the problem is jointly NP-hard and non-convex.

\section{Channel Assignment}

Since multiple users are multiplexed on the same channel, co-channel interference exist. We also know that NOMA is interference limited. Consequently, it is not practical for all users to perform NOMA jointly. Hence, channel assignment is necessary for users to perform NOMA.

3.1 Mosek Channel Assignment

The channel assignment is also solved using the latest edition of the cvx 2.0 mosek solver method 23,24 that effectively solves the linear binary problems. The solver output is a binary matrix indicating the preferences of users on the best-suited channel. 
3.2 Exhaustive Search Method (ESM)

Our aim is to obtain the best set of users to be multiplexed on the channel. Selection of all possible combination of users is possible through ESM where each channel accommodates every possible combination of the users. Due to exhaustive search, it is more complex and time consuming method but is an optimal approach.

We have assumed the notation of optimised user set $\mathcal{U}_{o p t}$ which consists of $U_{\text {opt }}$ number of users multiplexed on the $c$ th channel.

\section{Power Optimization}

After performing the channel assignment, we obtain a channel allocation list $\mathcal{L}_{u}$. Our next task is to find power allocation coefficient i.e. $a_{c, u}$ for known $\rho_{c, u}$ via optimization. Consequently, the optimization problem in 4 a for each channel boils down to

$$
\begin{array}{cl}
f\left(\boldsymbol{a}_{c}\right)=\max _{a_{c, u}} \sum_{u=1}^{U_{o p t}} \log _{2}\left(1+\frac{a_{c, u} p_{c} \mid h_{c, u}}{\eta+p_{c}\left|h_{c, u}\right|^{2}}\right. \\
\text { subject to } \\
\sum_{u=1}^{U_{o p t}} a_{c, u}=1 & \forall c \in \mathcal{L}_{u} \\
a_{c, u} \geq 0 & \forall c \in \mathcal{L}_{u}, \forall u \in \mathcal{U}_{o p t} \\
\left|h_{c, u-1}\right|^{2}\left(a_{c, u}-\sum_{v=1}^{u-1} a_{c, v}\right) \geq \frac{P_{t h} \eta}{p_{c}} & \forall u \in \mathcal{U}_{o p t} \\
\log _{2}\left(1+\gamma_{c, u}\right) \geq R_{c, u}^{m i n} & \forall c \in \mathcal{L}_{u}, \forall u \in \mathcal{U}_{o p t}
\end{array}
$$

where $\boldsymbol{a}_{c}=\left[a_{c, 1}, a_{c, 2}, a_{c, 3}, \cdots, a_{c, u}\right]$. It may be noted that in the SIC constraint, user index will run from the second user on each channel. In our NOMA system model, SIC is performed at the receiver in order to improve the channel gains. This is assured by allocating power in reverse order of the users' channel gain, i.e., if channel gains are in the order of $\left|h_{c, 1}\right|^{2}>\left|h_{c, 2}\right|^{2}$ then power allocation coefficients are taken as $a_{c, 1}<a_{c, 2}$. It is assumed that there shall be channel gain difference between two users for the occurrence of SIC 16. The worst situation for SIC when two users have same signal strength. In this scenario, SIC is not performed thereby causing large error propagation. Constraints (6d) and (6e) may respectively written as

$$
\begin{gathered}
p_{c}\left|h_{c, u-1}\right|^{2}\left(a_{c, u}-\sum_{v=1}^{u-1} a_{c, v}\right) \geq P_{t h} \eta \\
a_{c, u} p_{c}\left|h_{c, u}\right|^{2} \geq\left(2^{R_{c, u}^{m i n}}-1\right)\left(\eta+p_{c}\left|h_{c, u}\right|^{2} \sum_{v=1}^{u-1} a_{c, v}\right)
\end{gathered}
$$




\subsection{Programming}

The objective function formulated above in (6a) can be solved by using a global optimization method that is known as the difference of two convex functions or DC programming. The objective function defined in $6 \mathrm{a}$ can be rewritten in the form of $y\left(\boldsymbol{a}_{c}\right)-z\left(\boldsymbol{a}_{c}\right)$, where $y\left(\boldsymbol{a}_{c}\right)$ and $z\left(\boldsymbol{a}_{c}\right)$ are given as

$$
\begin{gathered}
y\left(\boldsymbol{a}_{c}\right)=\sum_{u=1}^{U_{o p t}} \log _{2}\left(\eta+p_{c}\left|h_{c, u}\right|^{2} a_{c, u}+p_{c}\left|h_{c, u}\right|^{2} \sum_{v=1}^{u-1} a_{c, v}\right) \\
z\left(\boldsymbol{a}_{c}\right)=\sum_{u=1}^{U_{o p t}} \log _{2}\left(\eta+p_{c}\left|h_{c, u}\right|^{2} \sum_{v=1}^{u-1} a_{c, v}\right) .
\end{gathered}
$$

It is found that both the functions in $(9)$ and $(10)$ are concave in nature with respect to $a_{c, u}$. Therefore, the function $\left[y\left(\boldsymbol{a}_{c}\right)-z\left(\boldsymbol{a}_{c}\right)\right]$ is a difference of convex functions 25]. The constraints defined in (7) and (8) are linear inequality. We now define a vector $\mathbf{e}_{m}^{(c)}$ corresponding to each $a_{c, u}$ for the $c$ th channel. For each channel, we have $n$ columns and $m$ rows. Each element of the above vector is defined as

$$
e_{m n}^{(c)}= \begin{cases}0 & \text { if } n=m \text { and } n<m \\ \frac{1}{\ln 2}\left|h_{c, u}\right|^{2} p_{c} & \text { if } n>m\end{cases}
$$

Now we define the gradient of $z\left(\boldsymbol{a}_{c}\right)$ at each $a_{c, u}$ as

$$
\nabla z\left(\boldsymbol{a}_{\boldsymbol{c}}\right)=\sum_{u=1}^{U_{o p t}} \frac{1}{\eta+p_{c}\left|h_{c, u}\right|^{2} \sum_{v=1}^{u-1} a_{c, v}} \boldsymbol{e}_{\boldsymbol{m}}^{(c)}
$$

The value of $a_{c, u}$ for each user is chosen such that it lies within the feasible region defined by the constraints $(6 \mathrm{~b})$ to $(6 \mathrm{e})$. The first order approximation of $z\left(\boldsymbol{a}_{c}\right)$ provides an upper bound on it, given as 25].

$$
z\left(\boldsymbol{a}_{\boldsymbol{c}}\right) \leq z\left(\boldsymbol{a}_{\boldsymbol{c}}^{(k)}\right)+\left\langle\nabla z\left(\boldsymbol{a}_{\boldsymbol{c}}^{(k)}, \boldsymbol{a}_{\boldsymbol{c}}-\boldsymbol{a}_{\boldsymbol{c}}^{(k)}\right\rangle\right.
$$

where $k$ denotes the iteration number. This provides a crucial and well approximated lower bound for the maximization problem as stated below in Lemma 1 followed by Lemma 2. All the constraints given in (6b) to (6e) are compact and continuous 6. Therefore, by Cauchy's theorem, the solution is always guaranteed to converge. Accordingly, when $\left|f\left(\boldsymbol{a}_{c}^{(k+1)}\right)-f\left(\boldsymbol{a}_{c}^{(k)}\right)\right| \leq \epsilon$ for very small $\epsilon$, it implies that the solution obtained in two successive iterations are very close, if not equal. It may then be assumed that the solution has converged.

$$
\begin{gathered}
\max _{\boldsymbol{a}_{c}} f\left(\boldsymbol{a}_{c}\right)=y\left(\boldsymbol{a}_{c}\right)-z\left(\boldsymbol{a}_{c}^{(k)}\right)-\left\langle\nabla z\left(\boldsymbol{a}_{c}^{(k)}\right), \boldsymbol{a}_{c}-\boldsymbol{a}_{c}^{(k)}\right\rangle \\
\text { s.t : }(6(\mathrm{~b}), 6(\mathrm{e}))
\end{gathered}
$$

Algorithm 1 given below returns stationary point $\boldsymbol{a}_{\boldsymbol{c}}^{*}$ for the objective $f\left(\boldsymbol{a}_{\boldsymbol{c}}^{(k)}\right)$. 


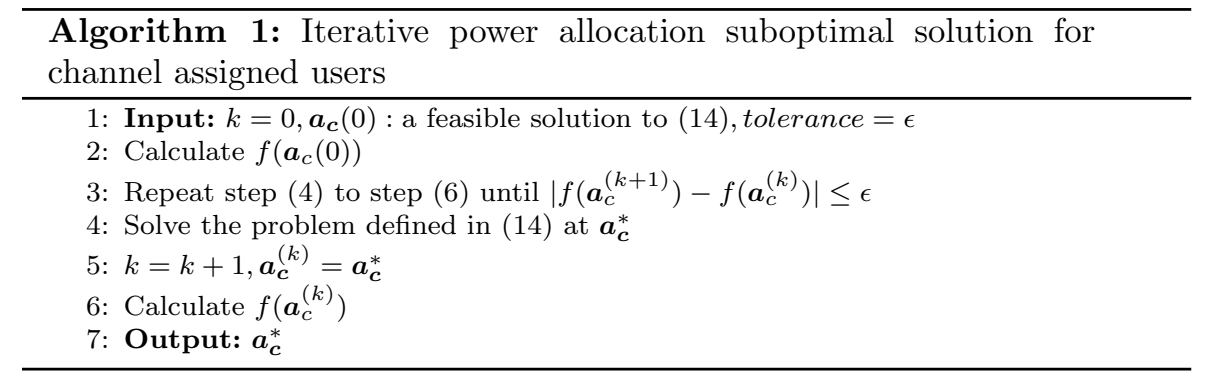

Lemma 1 Let $\omega$ be a differentiable function on a convex open set $a \subset \mathbb{R}$. Suppose that for every $s$ and $s^{\prime}$ in complex number $(M)$, we have $\omega(s)+\omega^{\prime}(s)\left(s^{\prime}-\right.$ $s) \geq \omega\left(s^{\prime}\right)$

Proof Let $\omega$ is concave in $\mathbb{C}$ and differentiable for all $s$ and $s^{\prime}$. From the definition of concavity, we have:

$$
\omega\left(\lambda s+(1-\lambda) s^{\prime}\right) \geq \lambda \omega(s)+(1-\lambda) \omega\left(s^{\prime}\right)-\omega\left(s^{\prime}\right) \forall s, s^{\prime} \in \mathbb{C},
$$

where $\lambda \in(0,1)$ Subtracting $\omega\left(s^{\prime}\right)$ from both the side of $(15)$, we get

$$
\begin{aligned}
& \omega\left(\lambda s+(1-\lambda) s^{\prime}\right)-\omega\left(s^{\prime}\right) \geq \lambda \omega(s)+(1-\lambda) \omega\left(s^{\prime}\right) \\
& \frac{\omega\left(\lambda s+(1-\lambda) s^{\prime}\right)-\omega\left(s^{\prime}\right)}{\lambda} \geq\left(\omega(s)-\omega\left(s^{\prime}\right)\right) \\
& \frac{\varphi(\lambda)-\varphi(0)}{\lambda} \geq \omega(s)-\omega\left(s^{\prime}\right)
\end{aligned}
$$

where $\varphi(\lambda)=\omega\left(\lambda s+(1-\lambda) s^{\prime}\right)$ and $\varphi(0)=\omega\left(s^{\prime}\right)$ at $\lambda=0$. From the differentiability property, we have

$$
\varphi^{\prime}(0)=\omega(s)-\omega\left(s^{\prime}\right)
$$

Also, we know that

$$
\begin{aligned}
\varphi^{\prime}(\lambda) & =\omega^{\prime}\left(\lambda s+(1-\lambda) s^{\prime}\right)\left(s-s^{\prime}\right) \\
\varphi^{\prime}(0) & =\omega^{\prime}\left(s^{\prime}\right)\left(s-s^{\prime}\right)
\end{aligned}
$$

From (17) and (18), we obtain

$$
\begin{aligned}
& \omega^{\prime}\left(s^{\prime}\right)\left(s-s^{\prime}\right) \geq \omega(s)-\omega\left(s^{\prime}\right) \\
& \omega^{\prime}\left(s^{\prime}\right)\left(s-s^{\prime}\right)+\omega\left(s^{\prime}\right) \geq \omega\left(s^{\prime}\right)
\end{aligned}
$$

The above lemma shows that the function $\omega$ is a concave and differentiable function.

Lemma 2 If $\boldsymbol{a}_{\boldsymbol{c}}(k)$ at $k=0$ is selected such that all the constraints given in (14) are satisfied then $f\left(\boldsymbol{a}_{c}, \boldsymbol{a}_{c}(k)\right)$ is a tight lower bound of $f\left(\boldsymbol{a}_{c}\right)$.

Proof $R_{c}\left(\boldsymbol{a}_{c}\right)=y\left(\boldsymbol{a}_{c}\right)-z\left(\boldsymbol{a}_{c}\right) \geq y\left(\boldsymbol{a}_{c}\right)-\left[z\left(\boldsymbol{a}_{c}(k)\right)+\left\langle\nabla z\left(\boldsymbol{a}_{c}(k)\right), \boldsymbol{a}_{c}-\boldsymbol{a}_{c}(k)\right\rangle\right]$. First inequality follows (14) therefore $f\left(\boldsymbol{a}_{c}\right) \geq f\left(\boldsymbol{a}_{c}, \boldsymbol{a}_{c}(k)\right)$. 


\subsection{Fractional Transmit Power Allocation (FTPA)}

Although it is simple to implement FTPA, it is unable to allocate resources optimally. FTPA scheme fails under limited resource availability and hence, maximization of system efficacy across multiplexed users is difficult. This scheme is unable to provide fairer power allocation coefficients to multiplexed users, Hence, it is a suboptimal approach [26]. The FTPA is described as follows

$$
p_{c, u}=\left\{p_{c} \frac{\left(\left|h_{c, u}\right|^{2}\right)^{-\beta}}{\sum_{i=1}^{U_{o p t}}\left(\left|h_{c, i}\right|^{2}\right)^{-\beta}}\right\} \quad \forall c \in C_{o p t}
$$

where $\beta$ is defined as decay the factor which is in the range 0 to 1 . The lower bound $\beta=0$ corresponds to equal power among multiplexed users on the same channel. Increasing value of $\beta$ corresponds to fair power allocation to users, i.e., weak user is associated with more power. So $\beta$ is an optimization parameter that is required to be calculated via simulation in such a way that the system performance is maximized. It may be noted that $\beta$ should be same for each channel.

\subsection{Computational Complexity}

The computational complexity analysis of the proposed method is carried out in two phases:

- Phase 1: If the number of channel is $C$ and $u$ is the number of users, out of $U$ total users, to be multiplexed on each channel and the total possible combination of user assignment to channel is $n$ then the complexity of ESM scheme for each combination is given as: $\left(\begin{array}{l}U \\ u\end{array}\right)+\left(\begin{array}{c}U-u \\ u\end{array}\right)+\left(\begin{array}{c}U-2 u \\ u\end{array}\right)+\ldots \ldots . .+\left(\begin{array}{l}u \\ u\end{array}\right)$. Therefore, the total complexity of optimal ESM is $n \sum_{b=1}^{C-1}\left(\begin{array}{c}U-b u \\ u\end{array}\right)$, which includes extensive search operation.

- Phase 2: Power allocation is done by DC programming scheme and its complexity is $\mathcal{O}\left(\left(\frac{U}{C}\right)^{3}\right)$. The complexity of SQP scheme is derived as $\mathcal{O}\left(\left(\frac{U}{C}\right)^{3} C\right)$. The computational complexity of IPM is given as $\gamma^{0.5}(\gamma+\theta) \theta^{2}$ [27], where $\gamma$ is interpreted as the number of inequality constraints and $\theta$ is defined as the number of variables. Therefore, the overall complexity of the problem is $\mathcal{O}\left(\left(\frac{U}{C}+1\right)^{0.5}\left(\frac{2 U}{C}+1\right)\left(\frac{U}{C}\right)^{2}\right)$.

\section{Simulation Results}

In order to determine the performance efficiency of the proposed resource allocation algorithm, we carried out experiments by Monte Carlo simulations, conducted on MATLAB R2020b platform. In our simulation experiment, BS is placed at the center of a circular region. The shortest distance between the 
TABLE 2

SIMULATION PARAMETERS

\begin{tabular}{cl}
\hline \hline Simulation Parameter & Value \\
\hline Cell radius & 300 meter \\
Path loss constant $(\zeta)$ & 2 \\
BS maximum transmit power & 2 watt to 20 watt \\
Noise power spectral density $\left(\sigma^{2}\right)$ & $-174 \mathrm{dBm} / \mathrm{Hz}$ \\
Number of active $U E(|\mathcal{U}|)$ & 9 \\
Number of channels $(|\mathcal{C}|)$ & 3 \\
Minimum cellular data rate $\left(R_{c, u}^{\min }\right)$ & $0.5 \mathrm{bps} / \mathrm{Hz}$ \\
Minimum power gap $\left(P_{\text {th }}\right)$ & $10 \mathrm{dBm}$ \\
Precision parameter $(\epsilon)$ & $10^{(-3)}$ \\
Circuit power consumption $\left(P_{\text {cir }}\right)$ & $20 \mathrm{dBm}$ \\
\hline \hline
\end{tabular}

$U E \mathrm{~s}$ is kept to be $30 \mathrm{~m}$ and also the minimum distance between $U E$ and the $\mathrm{BS}$ is maintained as $40 \mathrm{~m}$. The value of $\beta=0.9$. As we increase the value of $\beta$, it provides more power to poorer channel users. That is why, we have considered the value of $\beta$ near to one. The rest of the parameters are listed in Table 2 .

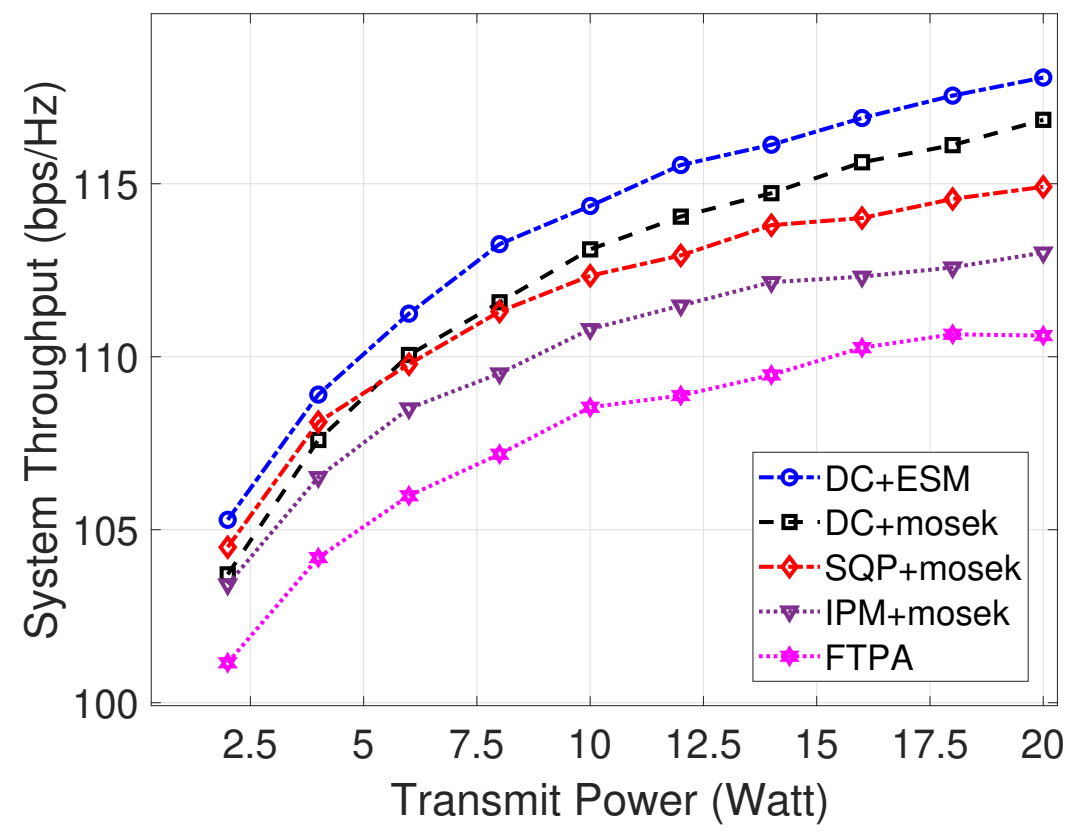

Fig. 2. Total achievable system throughput with respect to BS transmit power for , $|\mathcal{C}|=$ $3, U_{\text {opt }}=3, R_{c, u}^{\text {min }}=0.5 \mathrm{bps} / \mathrm{Hz}$ 
5.1 System Throughput versus Transmit Power

Fig. 2 depicts the feasible system throughput versus the BS transmission power. We observe that the system throughput increases as the available power budget of BS increases. It is noticed that the throughput achieved by a proposed scheme for the downlink NOMA system is better than SQP 8 and some other existing schemes 9. Moreover, the sum-rate gap between the proposed scheme and the exhaustive scheme is not significant enough. Further, the mean system throughput of the proposed scheme outperforms the existing schemes demonstrating the efficacy of proposed scheme. Furthermore, it is observed that the percentage of increase in the system throughput in our proposed scheme compared to that of the $\mathrm{SQP}+$ mosek scheme is $1.18 \%$ while the percentage decrease in throughput between the DC+ESM and DC+mosek (proposed) is $1.06 \%$

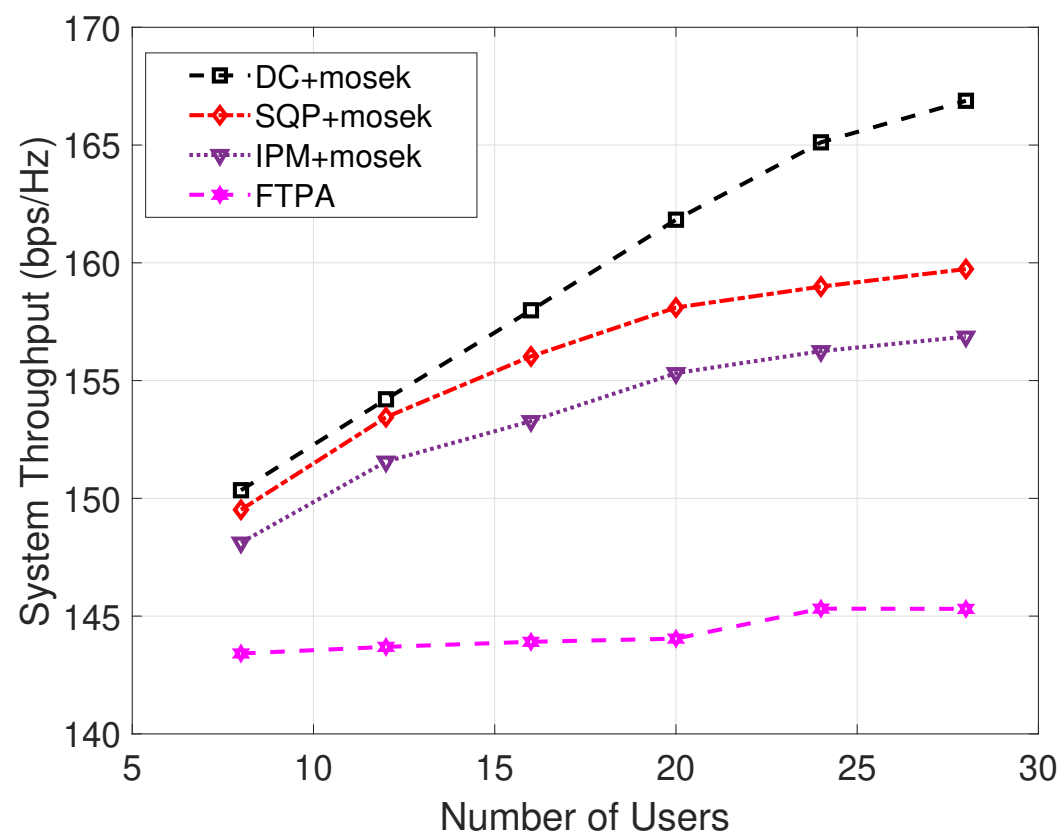

Fig. 3. Total achievable system throughput analysis with respect to $U E s, \beta=0.9, R_{c, u}^{\min }=$ $0.5 \mathrm{bps} / \mathrm{Hz},|\mathcal{C}|=3$

\subsection{System Throughput versus User}

Fig 3 shows that mean system throughput increases irrespective of the number of $U E$ s. Power allocation among users on each channel with DC programming 
gives better performance in comparison to the existing schemes as depicted in Fig.3. Simulation result also shows that how the number of $U E$ 's affects the downlink NOMA system throughput. System throughput increases with respect to the users. The reason behind increasing throughput is that $U E$ rates are getting added irrespective of the number of $U E$ s. Due to the high degree of complexity with respect to number of the users on each channel, we did not consider the ESM scheme for channel assignment. Additionally, if the number of users increases, the frequency reuse factor increases due to channel sharing of multiplexed users. As a result, the throughput of our system improved. Consequently, it shows that the efficacy of the proposed scheme with respect to interference handling capability degrades because interference increases as the frequency reuse increases due to accommodation of large number of users on the same radio resource. Our proposed scheme however performs better with increasing number of $U E \mathrm{~s}$. The throughput gap between the proposed scheme and the some other existing scheme increases at higher number of $U E$ by exploiting multiuser diversity. Also, the reason behind increasing system throughput is that $U E$ rates get added irrespective of the number of $U E$ s.

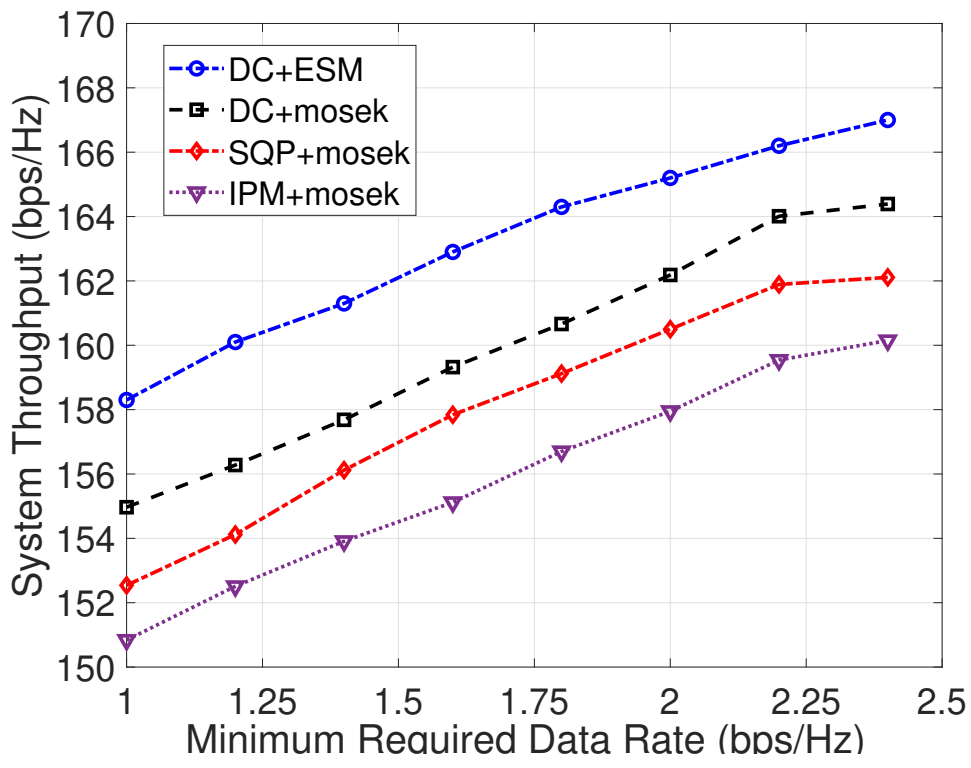

Fig. 4. Total achievable system throughput with respect to minimum required data rate of $U E, p_{c}=10$ watt, $|\mathcal{C}|=3, U_{\text {opt }}=3$,

\subsection{System Throughput versus QoS}

Fig. 4, illustrates the NOMA system throughput behavior at different QoS. It is observed that $U E$ throughput increases with increasing QoS. As the minimum 
required $U E$ data rate increases, more power is required by BS for transmission to achieve UEs' required data rate. It is also observed that the proposed scheme provides better performance as compared to a standard methods like IPM and SQP methods.

\subsection{Fairness Analysis by Jain Fairness Index}

Fairness is quantified by the fairness metric named Jain's fairness index (JFI). JFI determines normalized squared mean quantifying the degree of fairness, as given below

$$
J F I=\left\{\frac{\left(\sum_{u=1}^{|\mathcal{U}|} R_{c, u}\right)^{2}}{|\mathcal{U}| \sum_{u=1}^{|\mathcal{U}|} R_{c, u}^{2}}\right\}
$$

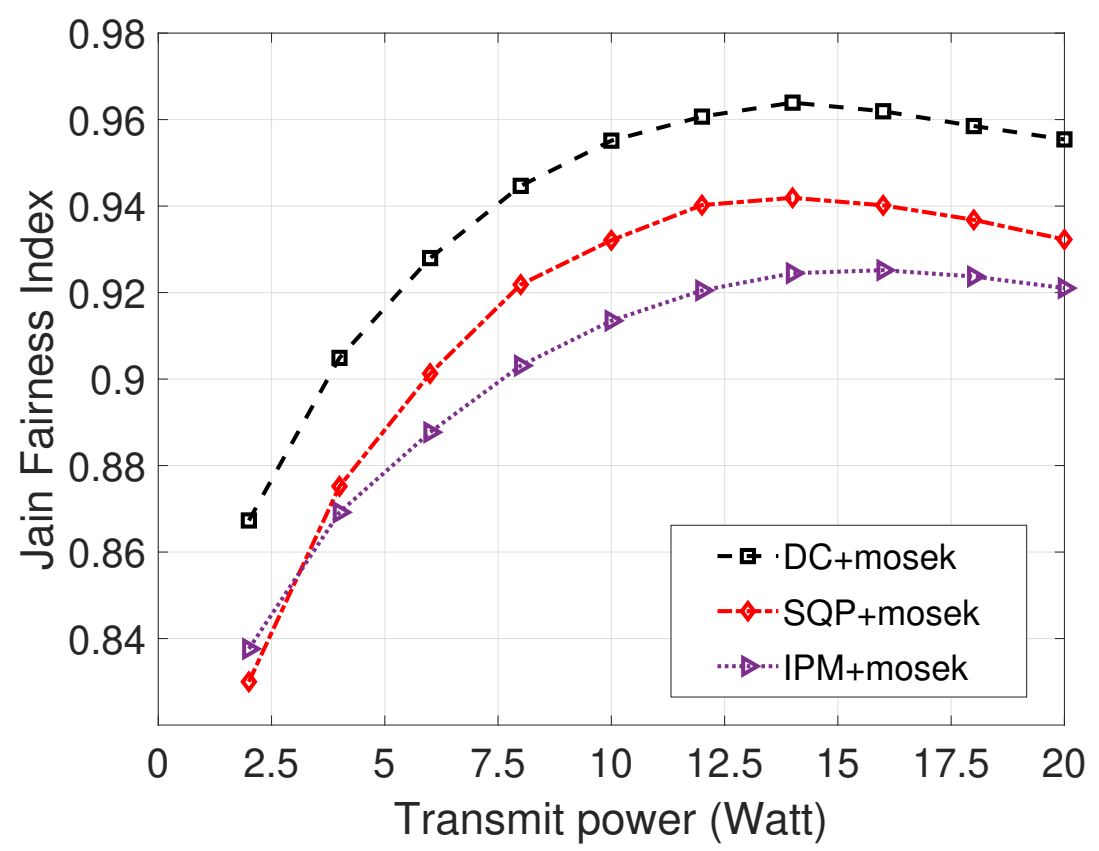

Fig. 5. Jain Fairness Index with respect to $\mathrm{BS}$ transmit power, $\beta=0.9, R_{c, u}^{\min }=0.5 \mathrm{bps} / \mathrm{Hz}$, $|\mathcal{C}|=3, U_{\text {opt }}=3$,

The range of JFI is $0<J F I<1$, where the lower bound corresponds to the minimum fairness of resource allocation and the upper bound corresponds to 
the maximum fairness of resource allocation. If the data rate of an individual user is close to each other at any instance, fairness is high and the value of JFI is also high. Hence, the contribution of individual data rate and system throughput is characterized by this fairness metric. From (21), we can say that as the sum of square of individual data rate is smaller, JFI will be more or, in other words, the scheme is fairer in terms of resource allocation w.r.t users. Fig. 5, shows that the JFI increases with respect to transmitted BS power budget and gradually tending to saturation indicating the fair distribution of resources among users. Thus, the resource distribution of the proposed scheme is fairer than some other existing schemes. The fairest resource distribution shows approximately the same throughput for each user. For example, if data rate of user A is $a$ and data rate of user B is $b$ at one channel then JFI is given as $\frac{(a+b)^{2}}{2 *\left(a^{2}+b^{2}\right)}$. According to the assumed scenario, suppose $\mathrm{a}=20 \mathrm{bps} / \mathrm{Hz}$ and $\mathrm{b}=19 \mathrm{bps} / \mathrm{Hz}$ then JFI will be 0.993 .

From Fig. 6, it may also be deduced that fairness indices of the proposed scheme decreases with respect to total number of users. In fact, other baseline scheme's performance also decreases but comparatively poorer than the proposed scheme due to increasing utilization of multiuser diversity.

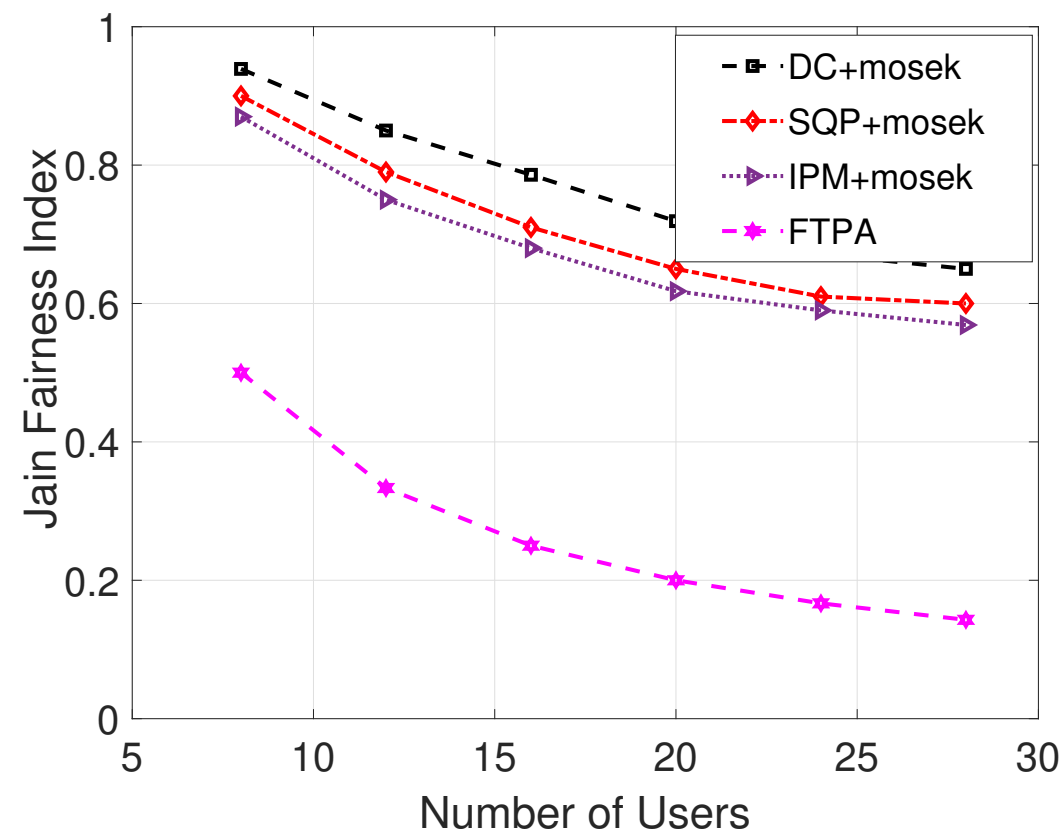

Fig. 6. Jain Fairness Index with respect to UE, $\beta=0.9, R_{c, u}^{\min }=0.5 \mathrm{bps} / \mathrm{Hz},|\mathcal{C}|=3$ 


\subsection{Energy Efficiency (EE) Analysis}

The best performance metric for analyzing power consumption is to calculate EE, since it is the ratio of sum-rate to the power consumption. Therefore, the reduction of power consumption increases the system EE [8]. it is mathematically defined as

$$
E E\left(a_{c, u}\right)=\frac{\sum_{c \in \mathcal{L}_{u}} \sum_{u=1}^{U_{o p t}} \mathrm{R}_{c, u}\left(a_{c, u}\right)}{\sum_{c \in \mathcal{L}_{u}} \sum_{u=1}^{U_{o p t}} a_{c, u} p_{c}+P_{c i r}}
$$

$P_{c i r}$ is the circuit power consumption and it is fixed to a constant value. Basically, EE is the ratio of total sum-rate and total power consumption.

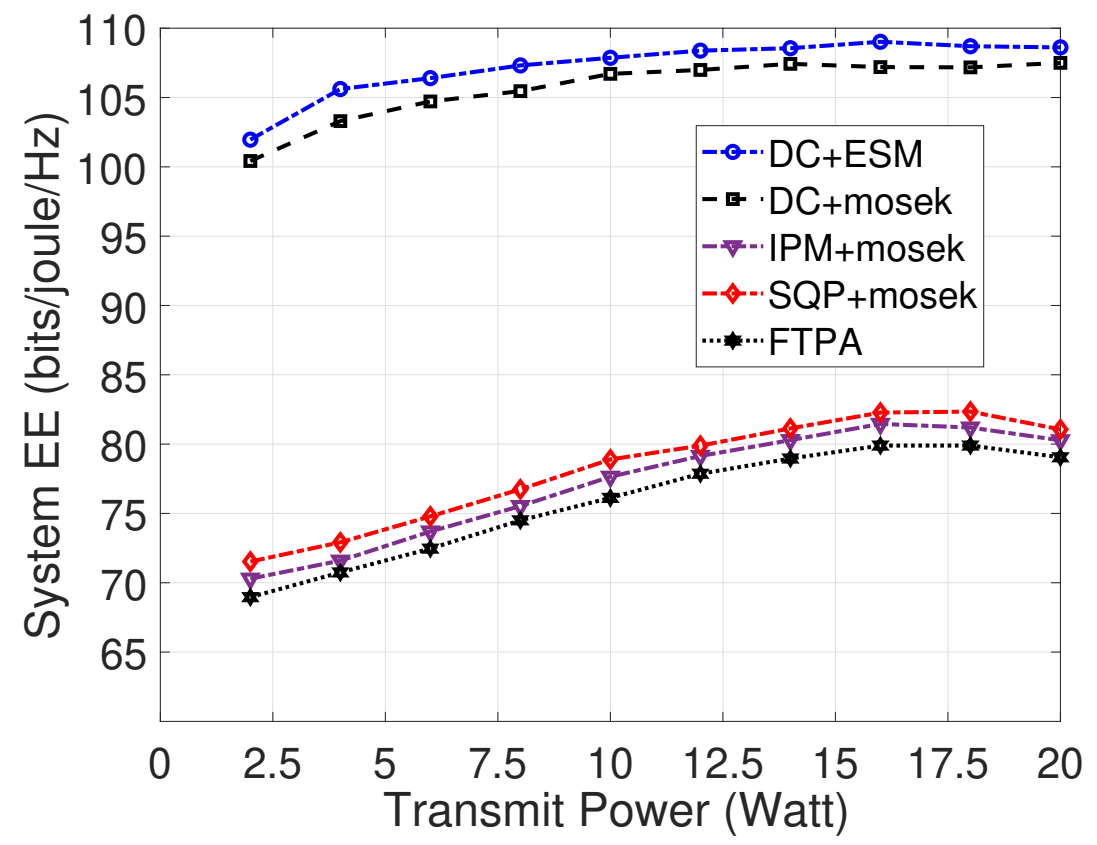

Fig. 7. Achievable system EE with respect to $\mathrm{BS}$ transmit power, $\beta=0.9, R_{c, u}^{\min }=0.5$ bps $/ \mathrm{Hz},|\mathcal{C}|=3, U_{\text {opt }}=3$

Sumrate is the summation of each UE's rate, which is a logarithmic function, and its rate of increase is slow with respect to the increase in BS power. System $\mathrm{EE}$ is plotted against transmitting power of BS in Fig. 7. It is observed that EE increases w.r.t BS power initially and reaches the saturation point but then decreases at a high power budget. Therefore, the proposed scheme 


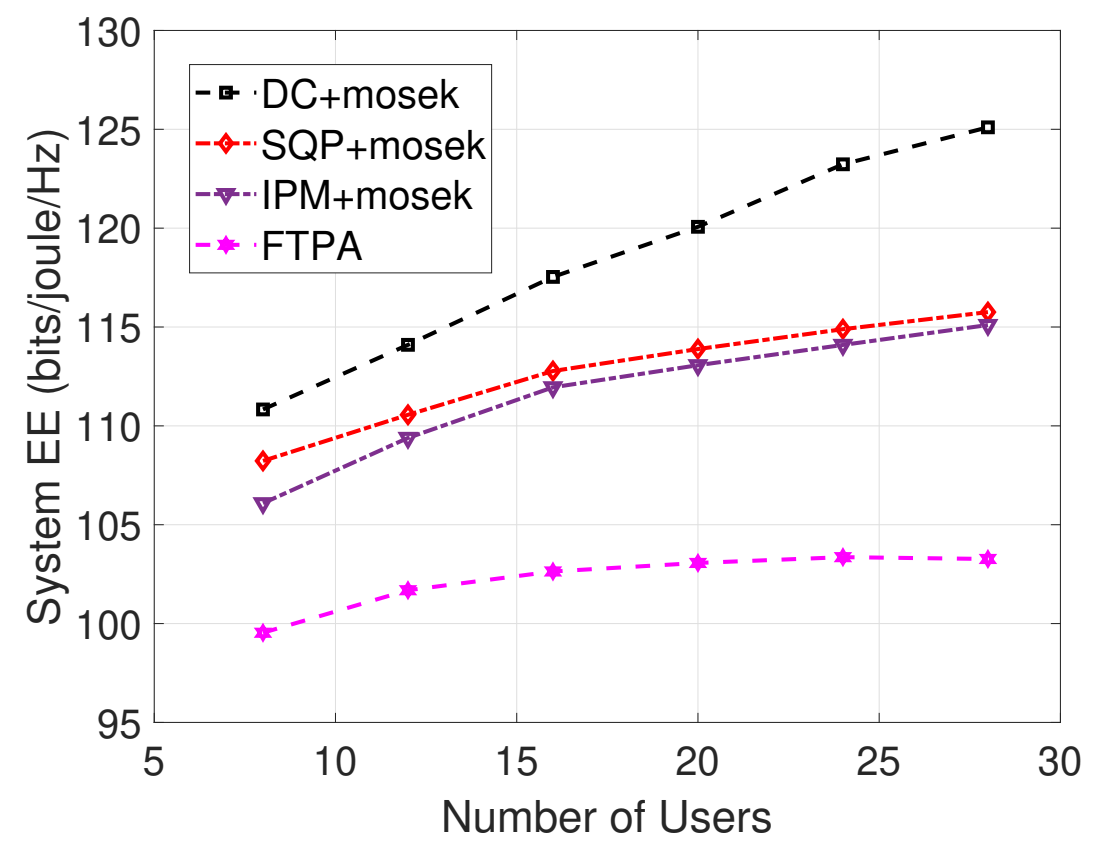

Fig. 8. Achievable system $\mathrm{EE}$ with respect to $\mathrm{BS}$ transmit power, $\beta=0.9, R_{c, u}^{\min }=0.5$ bps $/ \mathrm{Hz},|\mathcal{C}|=3, U_{\text {opt }}=3$

is more energy efficient than other existing schemes. In addition to that, our scheme gives better throughput at the cost of low power consumption from power budget.

System EE versus number of $U E$ is plotted in Fig 8 it is seen that the proposed scheme exceeds some other suboptimal schemes. As the number of users increases, the difference between the DC-based NOMA and SQP- based NOMA increases significantly whereas the performance of FTPA degrades with respect to the number of users that shows improper handling of the multiplexed users by the FTPA scheme.

\section{Conclusion}

In this paper, we have proposed an efficient throughput maximizaion scheme generalized for more than two users on the same channel that governs the wireless NOMA system. The proposed algorithm suggests a channel assignment technique and determines power allocation coefficients for multiplexed users. The channel assignment is done by mosek solver, while power is optimized by DC programming. This work considers joint channel and power allocation for $U E$ to maximize the total throughput of the users while assuring certain constraints such as the QoS requirement and SIC of $U E$. We formulated a joint 
optimization problem which however is NP-hard to solve. Therefore, we proposed an iterative solution approach for the same. As demonstrated through our simulation experiments, multiuser NOMA system successfully plays an important role in cancelling the interference effect on each channel. In future, we will consider multicell with different channel scenarios by considering more number of active users.

\section{References}

1. D. Tse and P. Viswanath, Fundamentals of Wireless Communications, 2004.

2. T. M. Cover and J. A. Thomas, Elements of Information Theory. Wiley-Interscience, 2006.

3. F.C Ribeiro, J. Guerreiro, R. Dinis, F. Cercas, D.N.K. Jayakody, Multi-user detection for the downlink of NOMA systems with multi-antenna schemes and power-efficient amplifiers, Physical Communication, vol. 33,pp. 199-205, 2019.

4. Z. Ali, Y. Rao, W.U. Khan, G.A.S Sidhu, Joint user pairing, channel assignment and power allocation in NOMA based CR systems, Applied Science, vol. 9,no.20, pp. 4282, 2019.

5. Z. Wei, J. Yuan,D.W.K.Ng, M.Elkashlan, and Z.Ding. "A survey of downlink nonorthogonal multiple access for 5G wireless communication networks", 2016.

6. P. Parida and S. S. Das, "Power allocation in OFDM based NOMA systems: A DC programming approach," 2014 IEEE Globecom Workshops (GC Wkshps), Austin, TX, pp. 1026-1031, 2014.

7. Z. Ding, M. Peng and H. V. Poor, "Cooperative Non-Orthogonal Multiple Access in 5G Systems," in IEEE Communications Letters, vol. 19, no. 8, pp. 1462-1465, Aug. 2015.

8. Khan, Wali Ullah, et al. "Efficient power allocation for NOMA-enabled IoT networks in 6G era." Physical Communication, 2020.

9. P. Gupta and D. Ghosh, "Channel Assignment with Power Allocation for Sum Rate Maximization in NOMA Cellular Networks," 2020 5th International Conference on Computing, Communication and Security (ICCCS), Patna, pp. 1-5, 2020.

10. J. Choi, "Non-Orthogonal Multiple Access in Downlink Coordinated Two-Point Systems," in IEEE Communications Letters, vol. 18, no. 2, pp. 313-316, February 2014.

11. Z. Ding, P. Fan and H. V. Poor, "Impact of User Pairing on 5G Nonorthogonal MultipleAccess Downlink Transmissions," in IEEE Transactions on Vehicular Technology, vol. 65, no. 8, pp, 2015.

12. Amin, Shahira H., et al. "Power allocation for maximum MIMO-NOMA system userrate." 2018 IEEE Globecom Workshops (GC Wkshps). IEEE, 2018.

13. Sun, Xiaofang, et al. "Joint beamforming and power allocation in downlink NOMA multiuser MIMO networks," in IEEE Transactions on Wireless Communications, 2018.

14. G. Liu, R. Wang, H. Zhang, W. Kang, T. A. Tsiftsis and V. C. M. Leung, "SuperModular Game-Based User Scheduling and Power Allocation for Energy-Efficient NOMA Network," in IEEE Transactions on Wireless Communications, vol. 17, no. 6, pp. 3877 3888 , June 2018 .

15. T. Manglayev, R. C. Kizilirmak and Y. H. Kho, "Optimum power allocation for nonorthogonal multiple access (NOMA)," 2016 IEEE 10th International Conference on Application of Information and Communication Technologies (AICT), Baku, pp. 1-4, 2016.

16. M. S. Ali, H. Tabassum and E. Hossain, "Dynamic User Clustering and Power Allocation for Uplink and Downlink Non-Orthogonal Multiple Access (NOMA) Systems," in IEEE Access, vol. 4, pp. 6325-6343, 2016.

17. S. Timotheou and I. Krikidis, "Fairness for Non-Orthogonal Multiple Access in $5 \mathrm{G}$ Systems," in IEEE Signal Processing Letters, vol. 22, no. 10, pp. 1647-1651, Oct. 2015.

18. J. Zhu, J. Wang, Y. Huang, S. He, X. You and L. Yang, "On Optimal Power Allocation for Downlink Non-Orthogonal Multiple Access Systems," in IEEE Journal on Selected Areas in Communications, vol. 35, no. 12, pp. 2744-2757, Dec. 2017. 
19. Z. J. Ali, N. K. Noordin, A. Sali, F. Hashim and M. Balfaqih, "An Efficient Method for Resource Allocation and User Pairing in Downlink Non-orthogonal Multiple Access System," 2019 IEEE 14th Malaysia International Conference on Communication (MICC), Selangor, Malaysia, pp. 124-129, 2019.

20. Z. Q. Al-Abbasi and D. K. C. So, "Power allocation for sum rate maximization in nonorthogonal multiple access system," 2015 IEEE 26th Annual International Symposium on Personal, Indoor, and Mobile Radio Communications (PIMRC), pp. 1649-1653, 2015.

21. Z. Q. Al-Abbasi and D. K. C. So, "User-Pairing Based Non-Orthogonal Multiple Access (NOMA) System," 2016 IEEE 83rd Vehicular Technology Conference (VTC Spring), pp. $1-5,2016$.

22. P. Gupta and D. Ghosh, "QoS Aware and Fair Resource Distribution for Uplink NOMA Cellular Networks," 2020 IEEE International Conference on Advanced Networks and Telecommunications Systems (ANTS), pp. 1-6, 2020.

23. Boyd, Stephen, Stephen P. Boyd, and Lieven Vandenberghe. Convex optimization. Cambridge university press, 2004.

24. E.D. Andersen and K.D. Andersen, "The MOSEK interior point optimizer for linear programming: an implementation of the homogeneous algorithm", High performance optimization. applied optimization, vol. 33, pp. 197-232, 2000.

25. H. H. Kha, H. D. Tuan and H. H. Nguyen, "Fast Global Optimal Power Allocation in Wireless Networks by Local D.C. Programming," in IEEE Transactions on Wireless Communications, vol. 11, no. 2, pp. 510-515, February 2012.

26. Y. Saito, A. Benjebbour, Y. Kishiyama and T. Nakamura, "System-level performance evaluation of downlink non-orthogonal multiple access (NOMA)," 2013 IEEE 24th Annual International Symposium on Personal, Indoor, and Mobile Radio Communications (PIMRC), London, pp. 611-615, 2013.

27. R. Gour and A. Tyagi, "Cluster oriented resource allocation and power optimisation for D2D network in cellular communications", IET Networks, vol. 9, no. 4, pp. 170-179, 7 2020 .

\section{Declarations}

\subsection{Funding}

"The authors declare that no funds, grants, or other support were received during the preparation of this manuscript."

7.2 Conflicts of interest/Competing interests

"The authors have no relevant financial or non-financial interests to disclose."

7.3 Availability of data and material (data transparency)

No data sets required in our work as it is totally simulation based work, therefore data are already generated through the simulation platform "MATLAB".

7.4 Code availability (software application or custom code)

Our custom code support the submitted claims. 
7.5 Authors' contributions

All the authors contributed equally in this research work. The first draft of the manuscript was written by Priya Gupta and both the authors commented on previous versions of the manuscript. All authors read and approved the final manuscript. 